\title{
Sperm Abnormalities: In the Male Partners of Infertile Couples from Kanyakumari District
}

\author{
Neena Viswambharan¹, Murugan Manavalan²
}

\begin{abstract}
Background: Infertility has been identified as a health issue worldwide and it is of great concern among married couples in our society. Scientifically, it has been established that both males and females can contribute to infertility. However, in most cases, women are held responsible for the same without proper diagnosis. Statistically, female factors contribute to about $40-60 \%$ of infertility cases, whereas $20-40 \%$ of infertility cases are due to factors contributed by males, of which, deficiency in semen quality is the major cause which accounts for $40-50 \%$ of infertility cases. A decrease in semen quality has been reported which can be due to lifestyle changes, occupational exposures to chemicals, heat, and stress. Aims: To study the prevalence of infertility, distribution of sperm abnormalities, and the impact of occupation on men visiting a tertiary clinic. Settings and design: Male partners of infertile couples who visited the fertility center were considered for the study.

Materials and methods: Seminal fluid was analyzed for volume, sperm concentration, motility (progressive motility), and morphology.

Results: In this study conducted in our center, we found that $55.8 \%$ of the patients had sperm abnormalities, among which teratozoospermia was the commonly observed anomaly.

Conclusion: Male factors equally contribute to infertility as female factors. Semen analysis is the crucial diagnostic test for infertility assessment of the male partner.

Key messages: The incidence of male infertility has been increasing recently and the evaluation and prognosis of male subfertility are challenging. Although semen analysis is the primary test for the assessment of infertility in males, it does not recognize the abnormality in idiopathic infertility. Thus, new techniques and methods need to be developed to improve accuracy and reduce variation.

Keywords: Infertility, Male, Motility, Semen.

International Journal of Infertility and Fetal Medicine (2020): 10.5005/jp-journals-10016-1199
\end{abstract}

\section{INTRODUCTION}

World Health Organization (WHO) and ICMART define infertility as a disorder of the reproductive system where a clinical pregnancy is not accomplished following a year or more of consistent unprotected sexual intercourse. ${ }^{1}$ In India, it is estimated that between 15 and 20 million couples encounter infertility annually and worldwide it ranges between 60 and 80 million couples for the same. A study by WHO, carried out from 1982 to 1985 , demonstrates that female factors contribute to $38 \%$ of infertility cases, male factors are responsible for $20 \%, 27 \%$ from both partners, and $15 \%$ are unexplained. According to a recent study, infertility resulting from male factors ranges between $40 \%$ and $50 \% .^{2}$ It is difficult to estimate the prevalence of male infertility mainly due to the patriarchal society in which a female partner is mostly held responsible for infertility. ${ }^{3}$ The factors contributing to male infertility can be due to biological factors, such as, testicular failure, cryptorchidism, genetic problems, or social factors, such as, contraceptive usage, stress, and age of marriage. ${ }^{4}$ Infertility can be categorized as primary infertility and secondary infertility. The former is a failure to achieve a successful pregnancy leading to live birth, whereas the latter is defined as the condition when a woman with a prior history of a successful pregnancy cannot bear a child. ${ }^{2}$ In India, the prevalence of primary infertility ranges from $3.9 \%$ to $16.8 \% .^{5}$

Based on various studies, the rate of infertility in India varies from state to state which ranges from 3.7\% in Uttar Pradesh and Himachal Pradesh, 3.5\% in Maharashtra, ${ }^{6}$ 5\% in Andhra Pradesh ${ }^{6}$ to $15 \%$ in Kashmir. ${ }^{7}$
${ }^{1}$ Department of Nanotechnology, Noorul Islam Centre for Higher Education, Kumaracoil, Tamil Nadu, India

${ }^{2}$ Department of Allied Health Science, Noorul Islam Centre for Higher Education, Kumaracoil, Tamil Nadu, India

Corresponding Author: Neena Viswambharan, Department of Nanotechnology, Noorul Islam Centre for Higher Education, Kumaracoil, Tamil Nadu, India, Phone: +91 9847033527, e-mail: neenaviswam@gmail.com

How to cite this article: Viswambharan N, Manavalan M. Sperm Abnormalities: In the Male Partners of Infertile Couples from Kanyakumari District. Int J Infertil Fetal Med 2020;11(1):16-19.

Source of support: Nil

Conflict of interest: None

Semen analysis, an essential procedure to assess male fertility, is the foremost fundamental assessment test that produces most of the data regarding testicular function, maturation of sperm, and functioning of the accessory glands. ${ }^{8}$

The present study is aimed at finding the incidence of different sperm abnormalities in men of infertile couples who visited for treatment in our center.

\section{Materials and Methods}

The current study includes a total of 1,460 couples who visited the center between June 2016 and May 2018 for evaluation and treatment of infertility. This study was conducted to find the

() The Author(s). 2020 Open Access This article is distributed under the terms of the Creative Commons Attribution 4.0 International License (https://creativecommons. org/licenses/by-nc/4.0/), which permits unrestricted use, distribution, and non-commercial reproduction in any medium, provided you give appropriate credit to the original author(s) and the source, provide a link to the Creative Commons license, and indicate if changes were made. The Creative Commons Public Domain Dedication waiver (http://creativecommons.org/publicdomain/zero/1.0/) applies to the data made available in this article, unless otherwise stated. 
prevalence of different sperm abnormalities and distribution of semen parameters in infertile men visiting our center.

Couples married for at least 12 months and having regular unprotected intercourse for 12 months were considered for the study. A detailed history of these couples was taken in a pro forma inclusive of age, marital status, years of marriage, family history, occupation, medical history, smoking, drinking, primary or secondary infertility, varicocele, surgical history for any illness, etc. Relevant investigations were conducted to rule out the female factor infertility and male partners of couples with female factor infertility were excluded from the study. Informed verbal consent was obtained from all the patients who were considered for the study. Patients were categorized into different age groups for the study. They were counseled regarding semen analysis as well as regarding the abstinence to be maintained for 2-7 days before semen analysis. Instruction for proper semen collection was provided before sample collection. The sample was collected by masturbation in a sterile wide-mouthed sample collection container and was kept in an incubator immediately. Semen analysis was performed as described in the WHO laboratory manual for the examination and processing of human semen, 5th Edition, 2010. ${ }^{9}$ The lower reference limit for normal semen analysis is given in Table 1.

Semen parameters, such as, volume, appearance, viscosity, $\mathrm{pH}$, sperm concentration, motility, morphology, and vitality, were determined. In the case of multiple semen analysis for the same patient, only the first result is taken for the study. The results were evaluated and categorized with reference as in Table 2.

\section{Results}

In the current study, the prevalence of male infertility was found to be $56.5 \%$. The incidence of primary infertility and secondary infertility found was $84.4 \%$ and $15.6 \%$, respectively.

Male patients considered for the study belonged to age groups of 26-43 years. A maximum number of patients belonged to the 31-35 years of age group followed by $36-40$ years, $26-30$ years, and the least number of patients belonged to the above 41 years of age group. Figure 1 represents the distribution of patients in different age groups.

Patients were categorized based on the findings from the semen analysis as follows:

Out of a total of 1,460 patients:

- Semen volume: 1,146 patients were observed with normal semen volume while 153 patients were with hyperspermia

Table 1: Lower reference limit of semen analysis

\begin{tabular}{ll}
\hline \multicolumn{2}{c}{ Semen analysis - WHO 2010* } \\
\hline Parameter & Lower reference limit \\
\hline Volume & $1.5 \mathrm{~mL}$ \\
$\mathrm{pH}$ & 7.2 \\
Sperm concentration & $>15 \mathrm{million} / \mathrm{mL}$ \\
Total sperm count & $39 \mathrm{million} /$ ejaculate \\
Total motility & $40 \%$ \\
Progressive motility (PR) & $32 \%$ \\
Morphology & $4 \%$ (strict criteria) \\
Vitality & $>58 \%$ \\
\hline
\end{tabular}

*WHO laboratory manual for the examination and processing of human semen (volume $>5 \mathrm{~mL}$ ) and 161 patients were with hypospermia (volume $<1.5 \mathrm{~mL}$ ).

- Sperm morphology: 645 patients were observed with normozoospermia with all parameters above the lower reference limit. 815 patients were observed with abnormalities in semen analysis, of which, 44 patients were azoospermic.

Table 2: Nomenclature in sperm quality*

\begin{tabular}{ll}
\hline Asthenozoospermia & Percentage of progressively motile \\
& sperm is below the lower reference \\
& limit
\end{tabular}

Asthenoteratozoospermia Percentages of progressively motile as well as morphologically normal sperm is below the lower reference limits

Azoospermia

Normozoospermia No sperm in the ejaculate

Total number of sperms with a percentage of progressively motile and morphologically normal sperm equals to or above the lower reference limits

Oligoasthenozoospermia Total number of sperms and percentage of progressively motile sperm below the lower reference limits

Oligoasthenoteratozoospermia (OAT)

Total number of sperms with percentages of progressively motile and morphologically normal sperm is below the lower reference limit. 5-14 million/ $\mathrm{mL}<4 \%$ morphology and progressive motility $<32 \%$

Oligoteratozoospermia Total number of sperms and percentage of morphologically normal sperm is below the lower reference limits

Oligozoospermia Total number of sperms is below the lower reference limit

Teratozoospermia Percentage of morphologically normal sperm is below the lower reference limit

Severe OAT

Total number of sperms $<5$ million $/ \mathrm{mL}$ with $<4 \%$ morphology and progressive motility $<32 \%$

Severe oligozoospermia Total number of sperms $<5$ million $/ \mathrm{mL}$

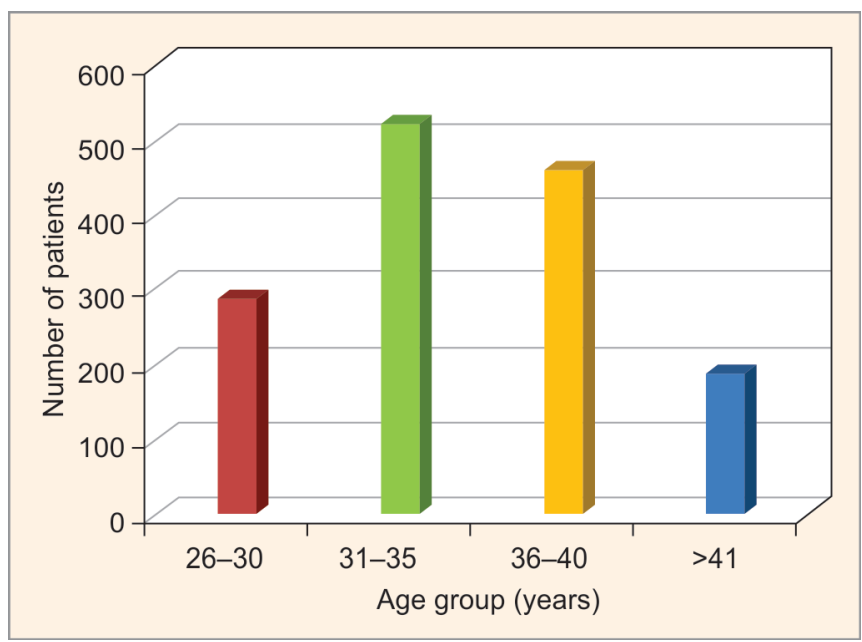

Fig. 1: Distribution of patients in the age group 
- Sperm vitality: 1,124 patients were observed with vitality $>58 \%$ (normal), whereas 336 patients were with vitality $<58 \%$.

The following graph summarizes the percentage of patients with normozoospermia and sperm abnormalities observed during our study (Fig. 2).

Furthermore, we categorized the patients based on their occupation. Predominantly patients with sperm abnormalities were from abroad (82.9\%) followed by patients working in the information technology (IT) field (78\%). A probable cause for this might be the exposure to extreme temperatures, stress, and imbalance in lifestyle. On the contrary, patients with the least percentage of sperm abnormalities found were in the Army (16\%) followed by laborers (21.3\%). Whereas mason (43.8\%), fishermen (57.6\%), and driver (58.8\%) had comparable sperm abnormalities. Table 3 demonstrates the categorization of patients based on their occupation.

\section{Discussion}

Semen analysis is a method to diagnose the fertility status of the male partner. Earlier, the females were considered to be the only factor for the cause of infertility. Sperm quality has a high influence on the rate of fertilization in assisted reproductive technology (ART) techniques. Sperm quality is based on count, motility, morphology, etc., assessed during semen analysis.

This study has been performed to identify the different sperm abnormalities observed in the patients who were coming for infertility treatment and also to identify whether occupation influences the sperm parameters.
There are very few reports available on the similar subject of male infertility from rural areas. The purpose of this study was to understand the prevalence of abnormalities in semen samples in the male partner of the infertile couples by examining the sperm concentration, motility, morphology, and vitality. In the current study, we categorized patients based on their occupation to understand which group of occupation experiences maximum abnormalities.

We considered 1,460 patients for this study and the prevalence of male infertility found was $56.5 \%$. This result is comparable to $52 \%$ and $54.33 \pm 2.08 \%$ as shown in a study by Bhaduri et al. and Sharmila et al. 2,10

In a report by $\mathrm{WHO}$, primary infertility ranges between $3.9 \%$ and $16.8 \%$ in India, ${ }^{5}$ whereas according to a study conducted by Zarger et al., it is $50 \%$. However, in the current study, we found primary infertility to be $84.4 \%$ which is comparable to $82.48 \%$ and $84.1 \%$ from a report by Shamila et al. and Juneja et al., respectively. 5,10 Majority of our patients belonged to $31-35$ years of age and $55.8 \%$ had abnormal spermiogram which is comparable to $52 \%$ and $56.38 \% .^{11,12}$

Our study showed that teratozoospermia (17.20\%) was the most common sperm abnormality. According to a study conducted by Juneja et al. in Assam and Jajoo et al. in rural central India, asthenozoospermia is the most commonly encountered sperm abnormality ${ }^{12,13}$ while in another study conducted in rural central India and Karnataka, oligozoospermia is the frequently observed sperm anomaly. ${ }^{14,15}$

While considering the effect of occupation on fertility, we found that patients from abroad followed by IT professionals were the

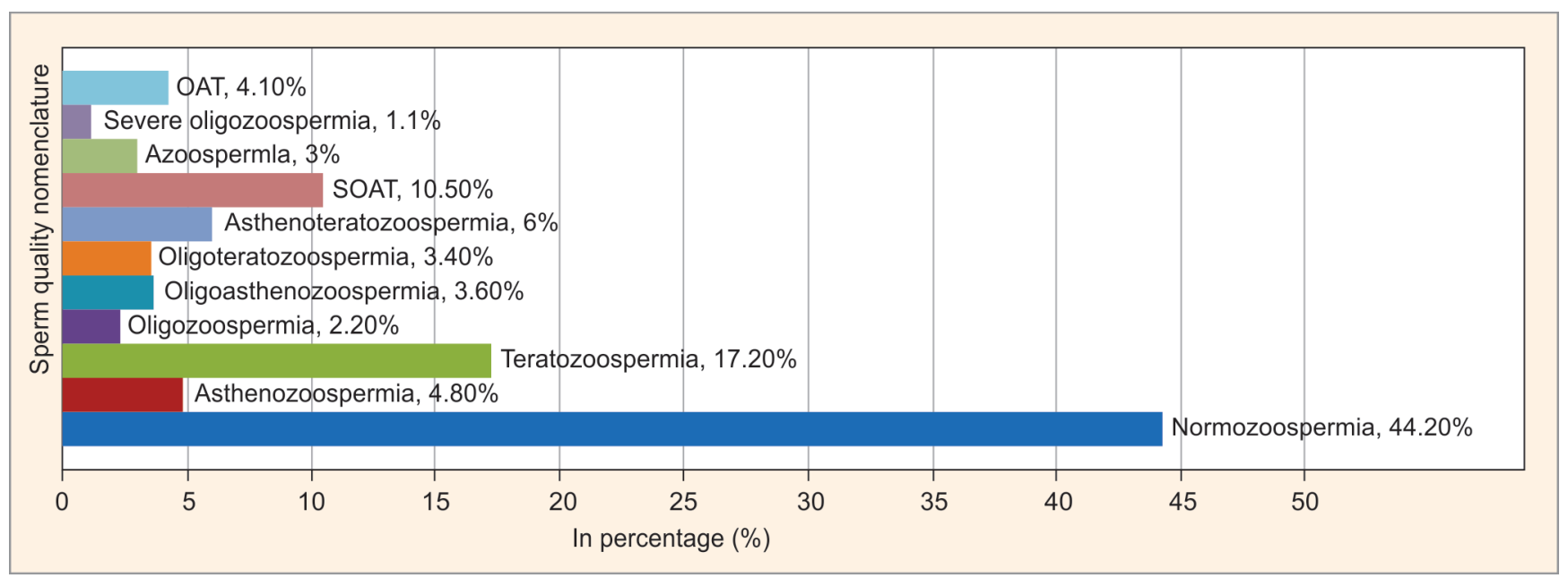

Fig. 2: Distribution of sperm analysis results

Table 3: Distribution of semen analysis result based on occupation

\begin{tabular}{lcclcl}
\hline Occupation of patient & No. of patients & Normozoospermia $(n)$ & Percentage & Abnormal $(n)$ & Percentage \\
\hline Mason & 347 & 195 & 56.2 & 152 & 43.8 \\
Abroad & 245 & 42 & 17.1 & 203 & 82.9 \\
Army & 25 & 21 & 84 & 4 & 16 \\
IT & 168 & 37 & 22 & 131 & 78 \\
Fisherman & 203 & 86 & 42.4 & 117 & 57.6 \\
Driver & 289 & 120 & 41.5 & 39 & 58.5 \\
Laborers & 183 & 144 & -615.7 & 815 & 21.3 \\
Total & 1,460 & 645 & - & \\
\hline
\end{tabular}


most affected and a major cause for this could be the pollution and lifestyle changes, such as, sedentary life, clothing, and posture. Continuous sitting and long hours of work such as for drivers and engineers will lead to increased scrotal temperature resulting in increased chances of infertility. ${ }^{16}$ People working abroad, in places, such as Saudi Arabia, Bahrain, and other Arab countries, are exposed to an extreme temperature which might be the cause for decreased sperm quality. On the contrary, patients who were working as laborers and soldiers were observed with better spermiogram results. This could be due to their active lifestyle.

\section{Conclusion}

In conclusion, the concept of clinical evaluation and management of both male and female partners are essential for effective infertility treatment. The prevalence of sperm abnormalities shows a different pattern in different studies within India. This difference might be due to regional ethnic, lifestyle, and environmental factors. There are many risk factors in an occupation that affect sperm quality and the impact of risk factors cannot be assessed individually as the exposure to each risk will be in different duration and severity. Thus, considering only one or two risk factors might result in underestimation of its consequence. An understanding and identification of the possible impact of risk factors in daily life are essential. Their impact could be reduced to a great extent by a change of lifestyle and necessary protective measures.

\section{References}

1. Agarwal $A$, Mulgund $A$, Hamada $A$, et al. A unique view on male infertility around the globe. Reprod Biol Endocrinol 2015;13:37. DOI: 10.1186/s12958-015-0032-1.

2. Bhaduri (Bhattacharyya) N, Sarkar AP, Dewasi N, et al. Abnormalities in semen analysis among male partners of infertile couples: a study in a tertiary care level hospital of West Bengal, India. Int J Reprod Contracept Obstet Gynecol 2015;4(1):100-102. DOI: 10.5455/23201770.ijrcog20150219.

3. Jajoo S, Kalyani KR. Prevalence of abnormal semen analysis in patients of infertility at a rural setup in Central India. Int J Reprod
Contracept Obstet Gynecol 2013;2(2):161-164. DOI: 10.18203/23201770.ijrcog20193785.

4. Joshi P, Gopal N, Bhat V. Study of semen analysis patterns in infertile males. Int. J Pharm Biol Sci 2011;1(1):44-49.

5. Juneja P, Phukan PK, Changmai D. A study of abnormal semen parameters in infertile couples in Assam, India. Int J Reprod Contracept Obstet Gynecol 2019;8(3):997-1000. DOI: 10.18203/23201770.ijrcog20190870.

6. Kumar N, Singh AK. Trends of male factor infertility, an important cause of infertility: a review of literature. J Human Reprod Sci 2015;8(4):191-196. DOI: 10.4103/0974-1208.170370.

7. Muhammad H, Ali Shah A, Nabi G, et al. Male infertility: etiological factors [a review]. Am Eur J Toxicol Sci 2015;7(2):95-103. DOI: 10.5829/ idosi.aejts.2015.7.2.9455.

8. Richard I. Lifestyle impact and the biology of the human scrotum. Reprod Biol Endocrinol 2007;5:15. DOI: 10.1186/1477-7827-5-15.

9. Rutstein SO, Shah IH, Infecundity, infertility, and childlessness in developing countries. DHS Comparative Reports No. 9. Calverton, MD, USA: ORC Macro and the World Health Organization, 2004.

10. Shamila S, Sasikala S. Primary report on the risk factors affecting female infertility in South Indian districts of Tamil Nadu and Kerala. Indian J Community Med 2011;36(1):59-61. DOI: 10.4103/09700218.80797.

11. Talwar PP, Go OP, Murali IN. Prevalence of infertility in different population groups in India and its determinants. Statistics and Demography. New Delhi: National Institute of Health \& Family Welfare \& Indian Council of Medical Research; 1986.

12. Unisa S. Childlessness in Andhra Pradesh, India: treatment-seeking and consequences. Reprod Health Matters 1999;7(13):54-64. DOI: 10.1016/S0968-8080(99)90112-X.

13. Vasan SS. Semen analysis and sperm function tests: How much to test? Indian J Urol 2011;27(1):41-48. DOI: 10.4103/0970-1591.78424.

14. World Health Organization. WHO Laboratory Manual for the Examination and Processing of Human Semen. 5th ed., Geneva: World Health Organization; 2010.

15. Zargar AH, Wani Al, Masoodi SR, et al. Epidemiologic and etiologic aspects of primary infertility in the Kashmir region of India. Fertil Steril 1997;68(4):637-643. DOI: 10.1016/s0015-0282(97)00269-0.

16. Zegers-Hochschild F, Adamson GD, de Mouzon J, et al. International Committee for Monitoring Assisted Reproductive Technology (ICMART) and the World Health Organization (WHO) revised glossary of ART terminology. Fertil Steril 2009;92(5):1520-1524. DOI: 10.1016/j. fertnstert.2009.09.009. 